\title{
Délibérations du Comité central de la FMH
}

Lors de sa séance du 21 octobre 2000, le Comité central (CC) s'est penché notamment sur les affaires suivantes:

\begin{abstract}
I. Assurances sociales
1. Nouveau modèle de rémunération des médicaments

Le 6 octobre 2000, le Département fédéral de l'intérieur (DFI) a fait connaître aux milieux médiatiques la décision du Conseil fédéral d'introduire au $1^{\mathrm{er}}$ janvier 2001 le nouveau modèle de rémunération pour les médicaments pris en charge par les caisses-maladie. Ce modèle prévoit que les prestations de conseils des pharmaciens et des médecins dispensants soit séparées des coûts effectifs des médicaments (fabrication et distribution) et rémunérées en fonction d'un tarif. Selon le DFI, la distribution de médicaments par les médecins propharmaciens devra être rémunérée par les prestations de base de la structure tarifaire TarMed.

Si ce modèle de rémunération entre réellement en vigueur le $1^{\text {er }}$ janvier 2001, douze mois s'écouleront encore jusqu'à l'introduction du TarMed et la rémunération des médicaments selon le TarMed, ce qui entraînera des diminutions de revenus dans les 13 cantons qui recourent à la propharmacie.

Le CC décide d'attendre la publication des évaluations de prix selon l'OFAS pour la liste des spécialités (LS). Lors de l'introduction du TarMed, il s'agira de prendre en considération les conséquences de la distribution des médicaments sur la neutralité des coûts. Le 14 décembre de cette année, la FMH organisera une session sur le thème de la remise directe des médicaments à laquelle M. Fritz Britt, vice-directeur de l’OFAS, sera invité à participer.
\end{abstract}

2. Recommandations d'honoraires pour les certificats médicaux dans le domaine de l'assurance privée (autrefois: "Liste tarifaire de la FMH») La "Liste tarifaire de la FMH» a été révisée la dernière fois le $1^{\text {er }}$ janvier 1995 et ne répond plus, depuis longtemps, à la réalité économique.

Une délégation du Comité central de la FMH, sous la direction du Dr R. Schwarz, président de la Société médicale de Bâle-Campagne, a revu cette liste de fond en comble avec les représentants de l'Association suisse d'assurances (ASA). Les nouvelles recommandations ont été adoptées par le Comité central de la FMH et l'ASA et mises en vigueur le $1^{\text {er }}$ novembre 2000.
Le secrétariat de la Commission de la concurrence, consulté préalablement, a émis de grandes réserves à l'égard de ces recommandations tarifaires. Il faut s'attendre à l'ouverture d'une enquête de droit cartellaire et, suivant ses résultats, dans le pire des cas, à l'interdiction du recourir aux recommandations d'honoraires.

Le CC relève que, s'agissant de recommandations et non de tarif obligatoire, nul n'est tenu d'y recourir. En conséquence, après information aux sociétés cantonales de médecine, lesdites recommandations seront publiées dans le BMS.

\section{Médecine du travail: conflit attendu entre} médecins de premier recours et sociétés de discipline médicale - démarches du côté de la FMH Jusqu'ici, les problèmes ont surtout concerné les dispositions transitoires de l'ordonnance sur les qualifications des spécialistes de la sécurité du travail. Dans ce domaine, un consensus a été trouvé pour l'essentiel entre les généralistes et la Société suisse de médecine du travail (SSMT) selon lequel les médecins qui, sans formation postgraduée spécifique, ont exécuté leur travail à la satisfaction de tous, ne doivent pas subir de tracasseries. Les pourparlers avec l'OFAS sont en cours et il n'y a pour l'instant aucune nécessité d'intervenir pour le CC.

Des problèmes pourraient cependant se poser à l'avenir. Lors d'une rencontre entre l'OFAS, le Secrétariat d'Etat à l'économie (SECO), le groupe «médecine du travail" de la CNA, la SSMT et la FMH, MM Kiener (SECO) et Schütz (CNA) se sont prononcés pour une voie des plus libérales à l'avenir. C'est ainsi que tous deux pensent qu'il vaut mieux - à l'avenir également - recruter sur place les médecins de premier recours montrant un véritable intérêt, quitte à ce qu'ils soient supervisés par un spécialiste de la médecine du travail. L'autre solution, celle de recruter à l'étranger les médecins du travail qui manquent en Suisse, ne serait pas favorable, à leur avis, à la qualité des soins. Mais les membres de la SSMT, quant à eux, sont convaincus que de nouvelles tâches dans le domaine de la médecine du travail ne doivent être assumées que par des spécialistes de cette discipline. C'est ainsi qu'un futur conflit avec les médecins de premier recours est quasiment programmé.

Le CC décide d'informer les médecins de premier recours du point de vue de la SSMT. Une première séance sous la direction d'une délégation du CC devrait réunir des représentants des médecins de premier recours et de la SSMT.

\section{Assurance-qualité}

1. Erreurs médicales - cas de décès iatrogènes Le plénum de la CFPC demande que l'on prenne énergiquement position contre les déclarations de l'OFAS de fin septembre dernier selon lesquelles, études étrangères à l'appui, 2000 à 3000 cas de décès par année seraient dus à des erreurs médicales. 
Le président informe que l'OFAS organise le 5 décembre 2000 une réunion sur les cas de mort iatrogène au cours de laquelle il tiendra un exposé. Les résultats et acquis devraient faire l'objet d'une réunion à plus large échelle sur le thème de «l'erreur médicale».

Selon le mot d'ordre «C'est de bons modes opératoires dont nous avons besoin, pas de mauvais médecins", le Comité central veut donner un sens positif à la chose et tirer parti des erreurs commises. Il s'agit de sensibiliser les membres de la FMH au problème sans recours aux manœuvres de dissimulation ou aux aveux de culpabilité. Le CC tient à nouveau à souligner l'importance de la formation continue avec recertification, une tâche qui relève de la CFPC.

\section{Formation prégraduée, postgraduée et continue}

1. Projet "perspectives formation postgraduée»: information des responsables

L'Assemblée plénière de la CFPC du 28 septembre 2000 a exprimé le vœu d'informer de manière exhaustive les responsables de la formation postgraduée sur le projet et d'organiser une enquête pour une formation de l'opinion préalable avant une prise de position de la FMH. Le président de l'assemblée a présenté le projet tout en souhaitant explicitement une consultation, malgré les mesures déjà prises dans plusieurs cantons ("médecins prestataires de service»).

Le CC décide de mener, auprès des responsables de la formation postgraduée, une consultation sur les perspectives en matière de formation postgraduée, d'en informer les sociétés cantonales de médecine et les sociétés de discipline médicale, avant d'ouvrir le débat sur l'internet.

2. Médecine physique et réadaptation: révision du programme de formation postgraduée; plus particulièrement: dispositions transitoires pour les responsables des cliniques de réadaptation Lors de la dernière révision du programme de formation postgraduée en médecine physique et réadaptation (entrée en vigueur le $1^{\mathrm{er}}$ janvier 2000), des dispositions transitoires spéciales ont été arrêtées qui devraient permettre aux responsables des cliniques de réadaptation d'obtenir plus facilement le titre de spécialiste FMH en médecine physique et réadaptation. A l'époque, le $\mathrm{CC}$ a donné son aval à la proposition de la société de discipline médicale à la condition, toutefois, que le titre de spécialiste ne soit octroyé qu'à la réussite de l'examen de spécialiste.

Dans plusieurs cas cependant, l'application de ces dispositions transitoires a conduit à des problèmes et, tout particulièrement, à des recours. Selon les directeurs de clinique concernés, la réussite de l'examen de spécialiste ne saurait être exigée, l'effet qualificatif de l'examen de spécialiste n'existant pas encore pour les candidats en formation postgraduée. La Société suisse de médecine physique et réadaptation demande dès lors de renoncer à l'attestation de la réussite de l'examen de spécialiste. Au nom de la société, le Dr Schneider propose subsidiairement que l'on exige un examen de spécialiste spécial, limité à la réadaptation.

Le CC soutient la proposition subsidiaire de la SSMPR.

\section{Organisation}

1. Election d'un nouveau président et vice-président pour le domaine des assistantes médicales

Dans le domaine des assistantes médicales, la question du laboratoire et des radiographies est régulièrement soulevée. Le CC souligne que les prestations de laboratoire doivent être exécutées par un personnel répondant aux exigences de qualités. Concernant le domaine radiologique, il se réfère à l'ordonnance sur la radioprotection qui contient en détail toutes les conditions nécessaires.

Le CC approuve le choix du Dr Michel Marchev, en tant que président, et du Dr Jürg Kremo, en tant que vice-président de la Commission des délégués aux questions relatives aux assistantes médicales.

Deutsch erschienen in Nr. 49/2000 